\title{
Pharmacological modulation of the voltage-gated neuronal Kv7/KCNQ/M-channel alters the intrinsic excitability and synaptic responses of pyramidal neurons in rat prefrontal cortex slices
}

\author{
Hui PENG ${ }^{1}$, Xi-ling BIAN ${ }^{2}$, Fu-cui MA ${ }^{2}$, Ke-Wei WANG ${ }^{2,3, *}$ \\ ${ }^{1}$ Department of Neurobiology, Neuroscience Research Institute, Peking University Health Science Center, Beijing 100191, China; \\ ${ }^{2}$ Department of Molecular and Cellular Pharmacology, State Key Laboratory of Natural and Biomimetic Drugs, Peking University School \\ of Pharmaceutical Sciences, Beijing 100191, China; ${ }^{3}$ Department of Pharmacology, Qingdao University School of Pharmacy, Qingdao \\ 266021, China
}

\begin{abstract}
The prefrontal cortex (PFC) critical for higher cognition is implicated in neuropsychiatric diseases, such as Alzheimer's disease, depression and schizophrenia. The voltage-activated Kv7/KCNQ/M-channel or M-current modulates the neuronal excitability that defines the fundamental mechanism of brain function. However, whether M-current functions to regulate the excitability of PFC neurons remains elusive. In this study, we recorded the native M-current from PFC layer V pyramidal neurons in rat brain slices and showed that it modulated the intrinsic excitability and synaptic responses of PFC pyramidal neurons. Application of a specific M-channel blocker XE991 $(40 \mu \mathrm{mol} / \mathrm{L})$ or opener retigabine $(10 \mu \mathrm{mol} / \mathrm{L})$ resulted in inhibition or activation of M-current, respectively. In the currentclamp recordings, inhibition of M-current was evidenced by the increased average spike frequency and the reduced first inter-spike interval (ISI), spike onset latency and fast afterhyperpolarization (fAHP), whereas activation of M-current caused opposite responses. Furthermore, inhibition of M-current significantly increased the amplitude of excitatory postsynaptic potentials (EPSPS) and depolarized the resting membrane potential (RMP) without affecting the miniature EPSC (mEPSC) frequency. These data demonstrate that voltage-gated neuronal Kv7/KCNQ/M-current modulates the excitability and synaptic transmission of PFC neurons, suggesting that pharmacological modulation of M-current in the PFC may exert beneficial effects on cognitive deficits implicated in the pathophysiology of neuropsychiatric disorders.
\end{abstract}

Keywords: Kv7; KCNQ; M-current; prefrontal cortex; pyramidal neurons; excitability; mEPSC; retigabine; XE991; neuropsychiatric diseases

Acta Pharmacologica Sinica (2017) 38: 1248-1256; doi: 10.1038/aps.2017.72; published online 12 Jun 2017

\section{Introduction}

The prefrontal cortex (PFC) is a key brain region involved in higher cognition, mnemonic and executive functions ${ }^{[1-3]}$. The neuronal activity of the PFC is critical for the formation of neural networks that define the fundamental functions of the brain, such as cognitive and emotional regulation and memory consolidation $^{[4-6]}$. PFC dysfunction is implicated in the pathophysiology of neuropsychiatric diseases, such as cognitive deficits, major depression and schizophrenia ${ }^{[7-12]}$.

Based on functional imaging studies, the PFC is the brain

\footnotetext{
${ }^{*}$ To whom correspondence should be addressed. E-mail wangkw@hsc.pku.edu.cn or wangkw@bjmu.edu.cn Received 2016-10-15 Accepted 2017-02-09
}

region most affected by the systemic administration of the $\mathrm{N}$-methyl-D-aspartate (NMDA) antagonists, producing a transient state of psychosis and schizophrenia-like cognitive deficits $^{[13,14]}$. Chronic stress causes structural remodeling and behavioral alterations in the PFC ${ }^{[15,16]}$. According to a postmortem study, a reduction in the medial PFC volume and/ or histopathological changes are involved in the pathogenesis of depressive symptoms ${ }^{[17]}$. Regulation of the intrinsic excitability or bursting of PFC neurons modulates cognitive behaviors such as fear conditioning tasks ${ }^{[18]}$ and partially restores memory-related firing of aged DELAY neurons to more youthful levels ${ }^{[8]}$. Therefore, functional modulation of neuronal activity in the PFC may lead to potential therapy for neuropsychiatric disorders. 
The Kv7/KCNQ/M-channels are a subfamily of voltagegated $\mathrm{K}^{+}$channels with five members, Kv7.1 to Kv7.5, encoded by KCNQ1-5 genes ${ }^{[19]}$. Slowly activating and non-inactivating M-channels are composed of Kv7.2 and Kv7.3 subunits in neurons of the central nervous system ${ }^{[20]}$. M-channels regulate neuronal excitability by controlling repetitive firing, action potential thresholds and the suppression of spontaneous firings ${ }^{[21]}$. The pre-synaptic localization of M-channels plays an important role in regulating glutamate release in the hippocampus $^{[22-24]}$. All these investigations suggest a critical role for the $\mathrm{Kv} 7 / \mathrm{KCNQ} / \mathrm{M}$-channel in regulating the excitability of PFC neurons ${ }^{[12]}$ and animal behaviors ${ }^{[25]}$. However, whether the function of the PFC is pharmacologically regulated by $\mathrm{Kv} 7 / \mathrm{KCNQ} / \mathrm{M}$-current modulators still remains elusive.

In the present study, we investigated the role of M-channel pharmacology in modulating excitability and synaptic response of pyramidal neurons in layer V of the PFC. Our findings show that M-current plays a critical role in modulating neuronal activity in the PFC, and pharmacological modulations of M-current may be beneficial for PFC dysfunction implicated in the pathophysiology of neuropsychiatric disorders, such as major depression and schizophrenia.

\section{Materials and methods}

\section{Slice preparations}

Male Sprague-Dawley rats (postnatal day 21-25) were deeply anesthetized with pentobarbital $(150 \mathrm{mg} / \mathrm{kg})$, and after decapitation, the brains were rapidly placed in ice-cold, oxygenated artificial cerebrospinal fluid (ACSF) containing (in mmol/L) $\mathrm{NaCl} 125, \mathrm{KCl} 2.5, \mathrm{NaH}_{2} \mathrm{PO}_{4} 1.25, \mathrm{NaHCO}_{3} 25, \mathrm{MgSO}_{4} 1, \mathrm{CaCl}_{2}$ 1.2 and glucose 16 (pH 7.2-7.4), which was bubbled with carbogen gas $\left(95 \% \mathrm{O}_{2}\right.$ and $\left.5 \% \mathrm{CO}_{2}\right)$. Coronal slices of the medial prefrontal cortex $(\mathrm{mPFC})(350 \mu \mathrm{m})$ were cut using a vibratome (VT 1200S, Leica), and brain slices were maintained at room temperature $\left(22-25^{\circ} \mathrm{C}\right)$ in a submerged-style holding chamber with oxygenated ACSF for at least $60 \mathrm{~min}$ before being transferred to the recording chamber perfused with oxygenated ACSF.

\section{Electrophysiological recordings}

Pyramidal neurons were visualized using infrared video microscopy with a $40 \times$ water-immersion objective (Nikon Instruments). Whole-cell patch clamp recordings were performed using glass pipettes with a resistance of 3-5 $\mathrm{M} \Omega$ when filled with internal solution containing (in mmol/L) $\mathrm{KCl} 20$, KGlu 125, HEPES 10, NaCl 4, EGTA 0.5, Mg-ATP 4, Tris-GTP 0.3 and phosphocreatine disodium salt hydrate 10 ( $\mathrm{pH}$ 7.2; 280-300 mOsm). Recordings were conducted in the soma of pyramidal neurons in layer $\mathrm{V}$ of the $\mathrm{mPFC}$, and cells were discarded when the access resistance exceeded $30 \mathrm{M} \Omega$. All recordings were low-pass filtered at $2.6 \mathrm{kHz}$ and acquired at $10 \mathrm{kHz}$ using MultiClamp 700B (Molecular Devices) and saved to a computer using DigiData 1440A and pClamp 10.0 (Molecular Devices).

Neurons were held at $-20 \mathrm{mV}$ for $1 \mathrm{~s}$ to activate the M-current before a deactivation step at $-40 \mathrm{mV}$ for $3 \mathrm{~s}$ for the current relaxation to record the native M-current. Changes in neuronal excitability were recorded by examining voltage responses to current inputs. A $1 \mathrm{~s}$ tonic step current in $20 \mathrm{pA}-$ increment step was injected into the soma to characterize the frequency-input curve. The first inter-spike interval (ISI) of the control condition was calculated from 5 repetitive spikes in the XE991 group, and 7 repetitive spikes in the retigabine and Q058L groups to determine their pharmacologic effects. The spike onset latency was calculated as the time from current input to the occurrence of the first spike. The fAHP was measured following a single spike in repetitive action potentials, and the value for the control condition was calculated from 5 repetitive spikes in all groups. Cells were voltage-clamped at $-65 \mathrm{mV}$ with a consistent perfusion of ACSF supplemented with bicuculline $(10 \mu \mathrm{mol} / \mathrm{L})$ and TTX $(0.5 \mu \mathrm{mol} / \mathrm{L})$ to record mEPSCs. Bipolar tungsten electrodes were placed in layer I of the $\mathrm{mPFC}, \sim 300 \mu \mathrm{m}$ from the recorded soma to evoke a single synaptic response. The current pulses (100-200 $\mu \mathrm{A}, 50 \mathrm{~ms})$ were applied through a constant current generator once every $20 \mathrm{~s}$. ACSF with bicuculline $(10 \mu \mathrm{mol} / \mathrm{L})$ was consistently applied.

\section{Chemicals}

Retigabine and QO58L were synthesized at Hebei Medical University ${ }^{[26]}$, and XE991 was purchased from Sigma.

\section{Data analysis and statistics}

Statistical significance was examined using unpaired Student's $t$ tests, where appropriate. The spike frequency at different injected currents was examined using two-way ANOVA, the cumulative probability of the mEPSC frequency was examined using a two-sample Kolmogorov-Smirnov test, and Chi-square statistics were used to investigate differences in the number of burst firing cells between the control condition and the XE991 group. A $P$-value $<0.05$ was considered statistically significant.

\section{Results}

\section{M-current recorded in layer V pyramidal neurons of the PFC}

We visually selected layer $\mathrm{V}$ pyramidal neurons in the PFC by identifying their large pyramidal-shaped somas $(\geq 20$ $\mu \mathrm{m}$ diameter $)^{[27]}$ and performed the whole-cell patch clamp recordings to identify the native M-current $\left(I_{\mathrm{M}}\right)$ (Figure $1 \mathrm{~A}$ and $1 \mathrm{~B})$. Using the characteristic voltage protocol that reveals $I_{\mathrm{M}}$ through its distinct voltage dependence and time course of deactivation that is distinguished from other $\mathrm{K}^{+}$ channels ${ }^{[28,29]}$, we recorded the $I_{\mathrm{M}}$ from layer $\mathrm{V}$ pyramidal neurons in slices of the PFC. The neurons were held at -20 $\mathrm{mV}$ for $1 \mathrm{~s}$ to activate the M-current before a deactivation step at $-40 \mathrm{mV}$ for $3 \mathrm{~s}$ to record a slowly relaxing outward current with time-dependent deactivation kinetics (Figure 1C). When the M-channel-selective blocker XE991 (40 $\mu \mathrm{mol} / \mathrm{L})$ was applied ${ }^{[20]}$, the characteristic $I_{\mathrm{M}}$ was substantially reduced, with a downward shift of the outward baseline current $\left(I_{\text {hold }}\right)$ (Figure 1D, top panel). The XE991-sensitive current was obtained by subtracting the XE991-resistant com- 
A

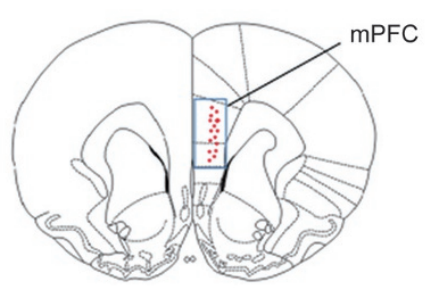

Bregma $2.20 \mathrm{~mm}$

D
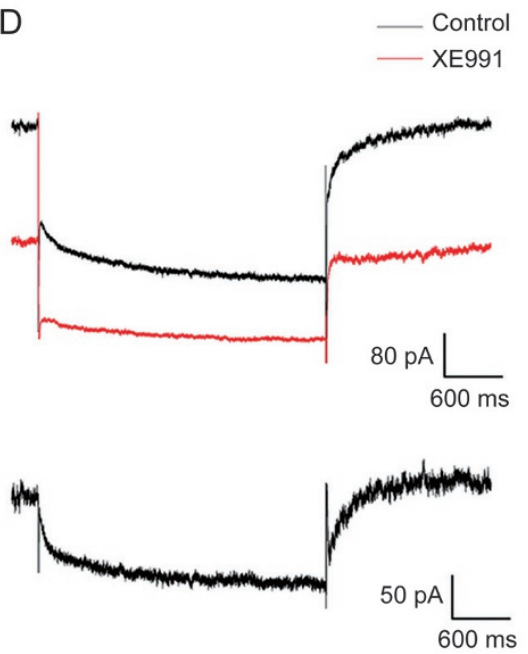

B

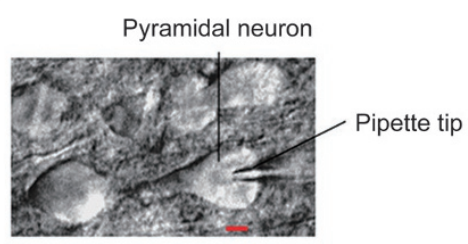

E
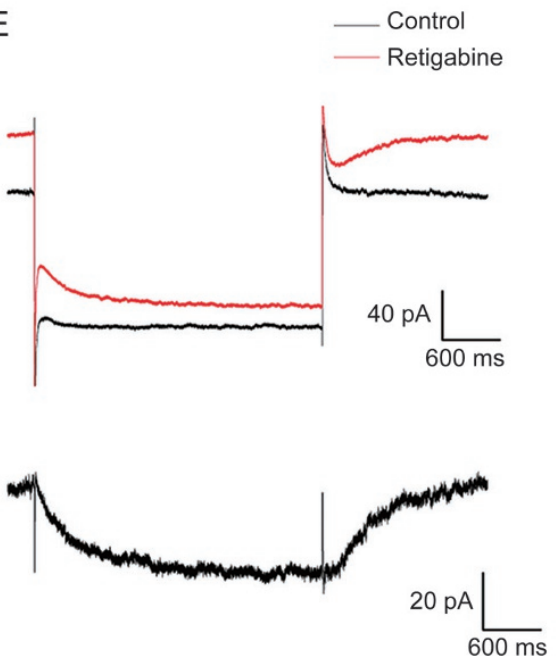

C

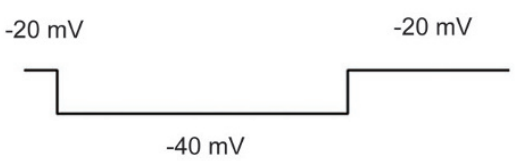

$\mathrm{F}$
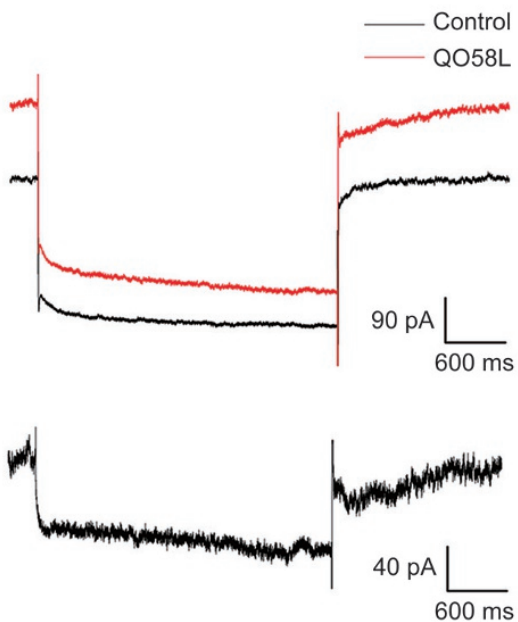

Figure 1. M-current recorded from PFC layer V pyramidal neurons. (A) Diagram showing the location of layer $\mathrm{V}$ pyramidal neurons in the medial PFC. (B) Representative image of whole-cell patch-clamp recording of PFC layer V pyramidal neurons. Scale bar, $10 \mu \mathrm{m}$. (C) Voltage-step protocol used to measure the M-current. (D) Top panel, representative traces showing the reduction of the M-current induced by XE991 (40 $\mu$ mol/L); bottom, the XE991sensitive current was determined by digitally subtracting the XE991-resistant component from the control current. (E) Top panel, representative traces showing the increase in the M-current induced by retigabine $(10 \mu \mathrm{mol} / \mathrm{L})$; bottom, the increased M-current was determined by subtracting the control current from the retigabine-enhanced component. (F) Top panel, representative traces showing the increase in the M-current induced by the opener Q058L (10 $\mathrm{\mu mol} / \mathrm{L})$; bottom, the increased M-current was determined by subtracting the control current from the Q058L-augmented component.

ponent from the control current (Figure 1D, bottom). We also applied a $10 \mu \mathrm{mol} / \mathrm{L}$ dose of the $\mathrm{M}$-channel-selective opener retigabine to further verify the recorded native $I_{\mathrm{M}}{ }^{[30]}$ and recorded an increase in the $I_{\mathrm{M}}$ in the presence of retigabine along with an upward shift of $I_{\text {hold }}$ (Figure 1E, top panel). The retigabine-enhanced current was obtained by subtracting the control current from the increased current (Figure 1E, bottom). Because retigabine has been reported to not only open M-channels but also to increase GABAergic IPSCs via a postsynaptic interaction with $\mathrm{GABA}_{\mathrm{A}}$ receptors ${ }^{[31]}$, we selected another M-channel opener, QO58-Lysine (QO58L). QO58 has recently been characterized as an effective M-channel opener with good selectivity for Kv7.2 and Kv7.4 ${ }^{[32]}$. Similarly, QO58L $(10 \mu \mathrm{mol} / \mathrm{L})$ increased the native $I_{\mathrm{M}}$ with an upward shift of $I_{\text {hold }}$ (Figure $1 \mathrm{~F}$ ). Taken together, the results obtained from the experiments using three different M-channel modulators revealed the presence of $I_{\mathrm{M}}$ in PFC layer $\mathrm{V}$ pyramidal neurons.

Table 1. The effect of Kv7/KCNQ/M-channel modulators XE991, retigabine and Q058L on membrane properties of PFC layer V pyramidal neurons.

\begin{tabular}{llcr}
\hline & Resting membrane potential $\left(V_{\mathrm{m}}, \mathrm{mV}\right)$ & Input resistance $\left(R_{\text {in }}, \mathrm{M} \Omega\right)$ & mAHP amplitude $(\mathrm{mV})$ \\
\hline Control & $-69.1 \pm 0.9(n=10)$ & $145.5 \pm 12.1(n=10)$ & $3.5 \pm 0.2(n=10)$ \\
XE991 & $-64.2 \pm 1.1^{* *}(n=10)$ & $165.8 \pm 11.9(n=10)$ & $3.4 \pm 0.3(n=10)$ \\
Control & $-62.1 \pm 1.3(n=7)$ & $207.9 \pm 17.6(n=7)$ & $2.9 \pm 0.4(n=7)$ \\
Retigabine & $-62.2 \pm 1.4(n=7)$ & $217.4 \pm 27.8(n=7)$ & $4.9 \pm 0.7^{*}(n=7)$ \\
Control & $-66.8 \pm 1.3(n=10)$ & $218.4 \pm 28.5(n=10)$ & $3.6 \pm 0.3(n=10)$ \\
Q058L & $-66.1 \pm 1.5(n=10)$ & $186.3 \pm 35.0(n=10)$ & $3.0 \pm 0.3(n=10)$ \\
\hline
\end{tabular}

Resting membrane potential $\left(V_{\mathrm{m}}\right)$, input resistance $\left(R_{\text {in }}\right)$, and mAHP amplitude of PFC layer $\mathrm{V}$ pyramidal neurons in the control condition and in the presence of XE991 (40 $\mu \mathrm{mol} / \mathrm{L})$, retigabine $(10 \mu \mathrm{mol} / \mathrm{L})$ and Q058L $(10 \mu \mathrm{mol} / \mathrm{L})$. Data are presented as mean \pm SEM. $n=7-10$. * $P<0.05,{ }^{* *} P<0.01$. 
A
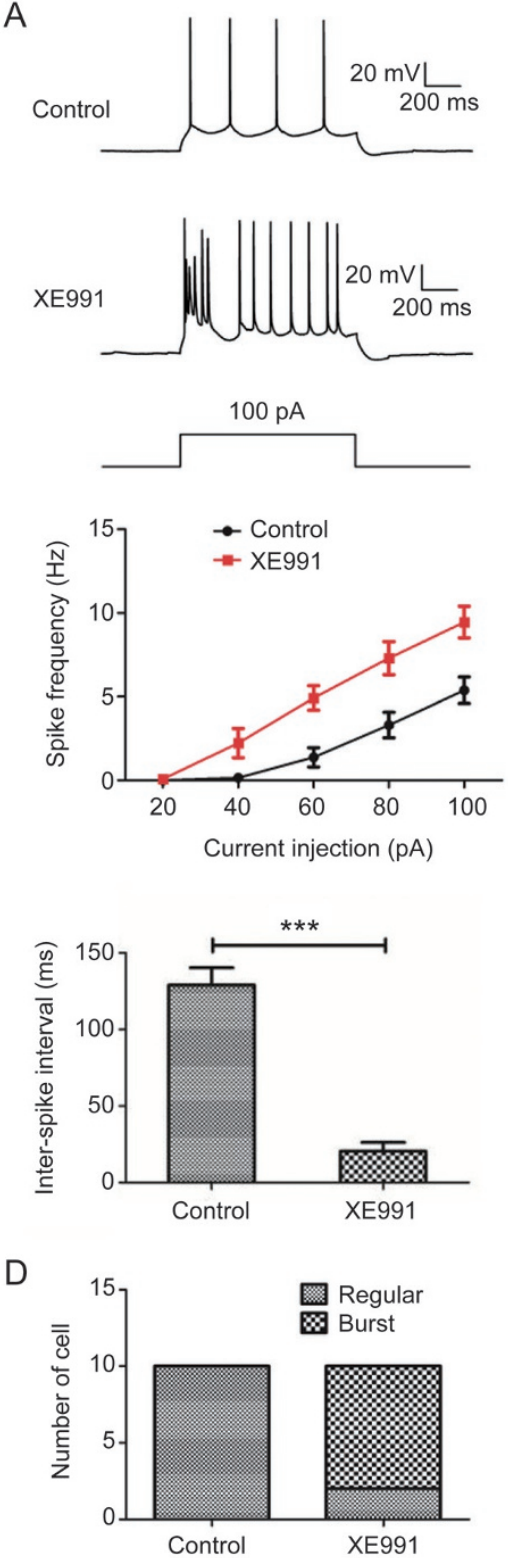

B
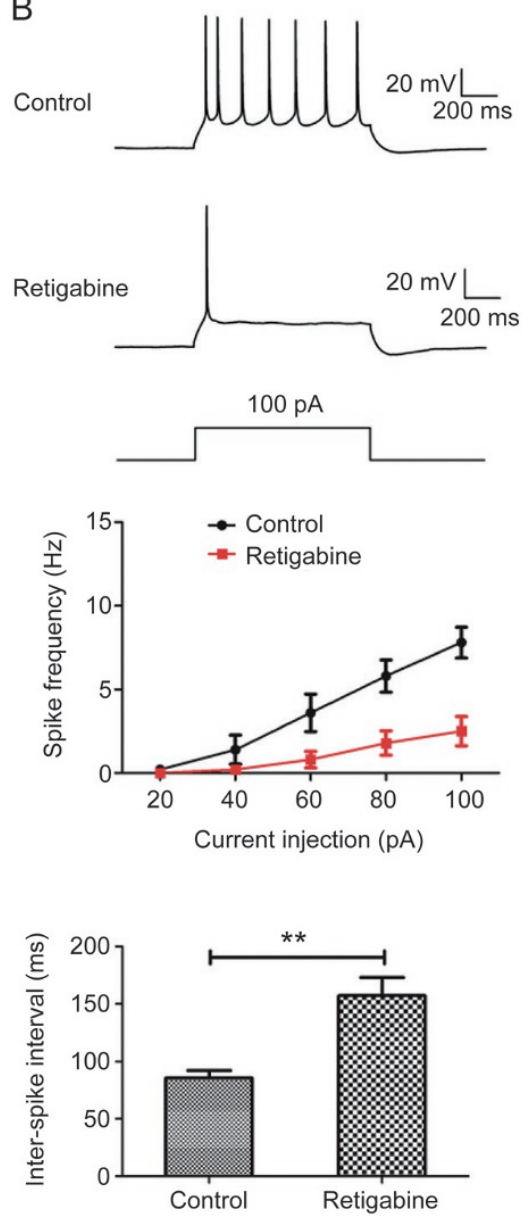

C
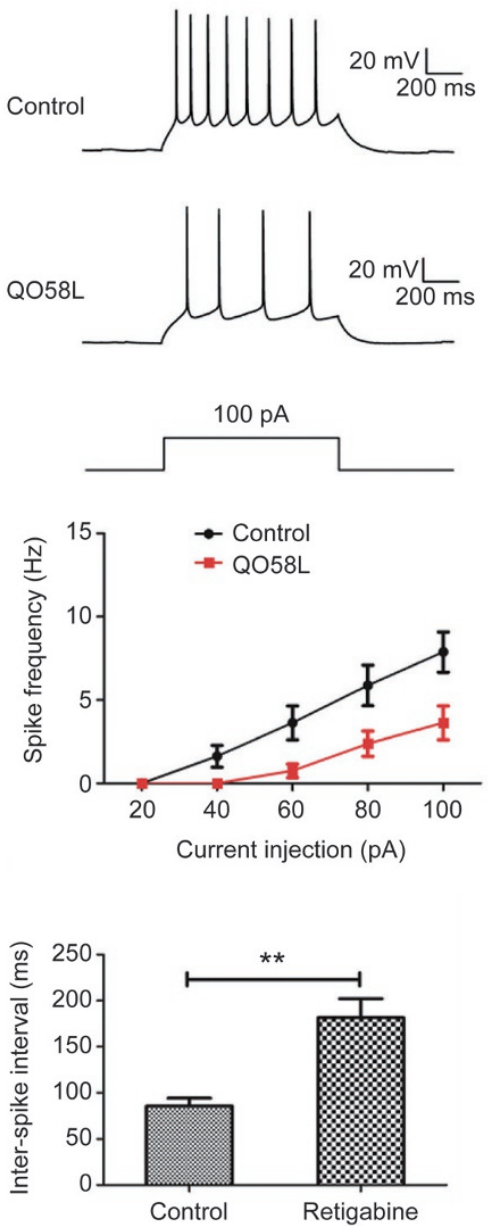

Figure 2. The M-current modulated the intrinsic excitability of PFC layer $V$ pyramidal neurons. (A) Top panel, representative traces of action potentials evoked by a 100 pA step current before and after the application of XE991 (40 $\mu \mathrm{mol} / \mathrm{L})$; note the burst firing. Middle, graphs illustrating the statistically significant increase in the average spike frequency evoked at different injected currents induced by XE991; bottom, bar graph indicating the XE991induced reduction of the ISI. The ISI of control condition was calculated from 5 repetitive spikes. (B) Top panel, representative traces of action potentials evoked by a $100 \mathrm{pA}$ step current before and after the application of retigabine $(10 \mu \mathrm{mol} / \mathrm{L})$. Middle, graphs illustrating the statistically significant reduction in the average spike frequency evoked at different injected currents induced by retigabine; bottom, bar graph indicating the retigabine-induced increase in the ISI. The ISI of the control group was calculated from 7 repetitive spikes. (C) Top panel, representative traces of action potentials evoked by a $100 \mathrm{pA}$ step current before and after the application of Q058L (10 $\mathrm{mmol} / \mathrm{L})$. The middle panel illustrates the reduction in the average spike frequency evoked at different injected currents induced by Q058L; bottom panel, bar graph indicating that the ISI was increased by Q058L. The ISI of the control group was calculated from 7 repetitive spikes. (D) Bar graph showing the XE991-induced increase in the number of burst firing cells compared with the number of regular firing cells in the control. Data are presented as the mean \pm SEM. $n=5-13 .{ }^{* *} P<0.01,{ }^{* * *} P<0.001$.

Modulation of the intrinsic excitability of PFC layer $\mathrm{V}$ pyramidal neurons by M-current modulators

To investigate the role of M-channels in regulation of intrinsic excitability of PFC layer V pyramidal neurons, we assessed the effect of M-channels on firing. The membrane properties were measured before and after the application of the three modu- lators (Table 1). XE991 significantly depolarized the resting membrane potential (RMP) $(n=10, P<0.01)$, without any effect on $R_{\text {in }}(n=10$, all $P>0.05)$. Both openers, retigabine and QO58L, had no influence on RMP and $R_{\text {in }}$ (retigabine, $n=7$; QO58L, $n=10, P>0.05)$. We examined two parameters, spike frequency and inter-spike interval (ISI), to measure intrinsic neuronal 
A
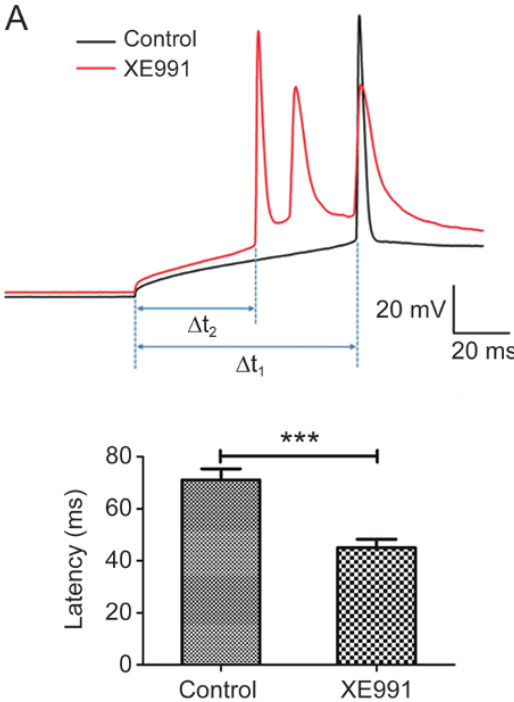

D
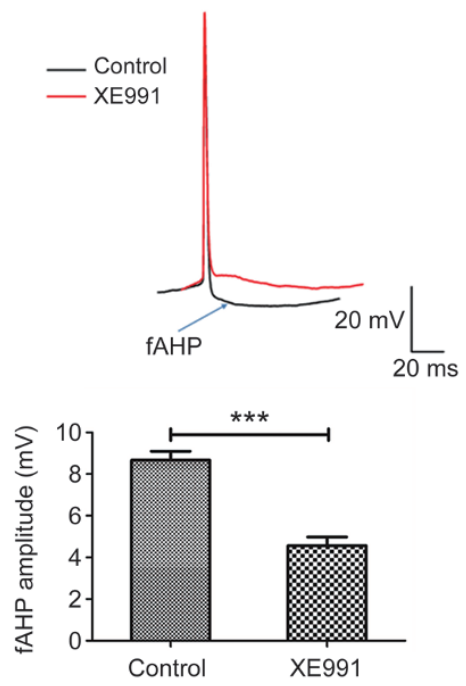

B
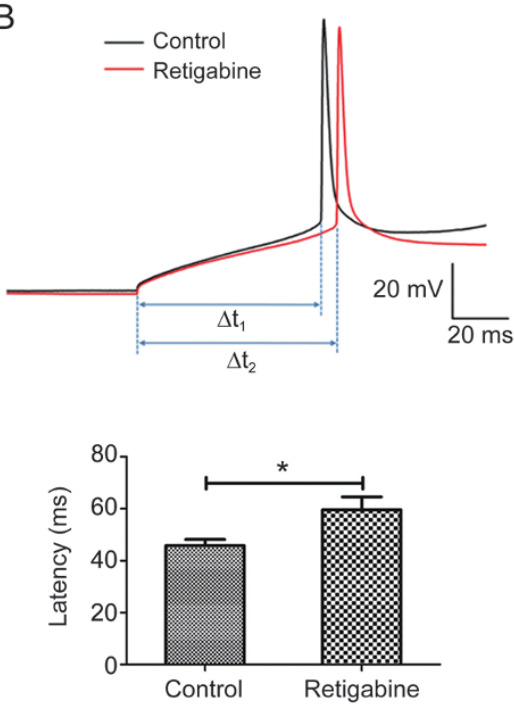

E
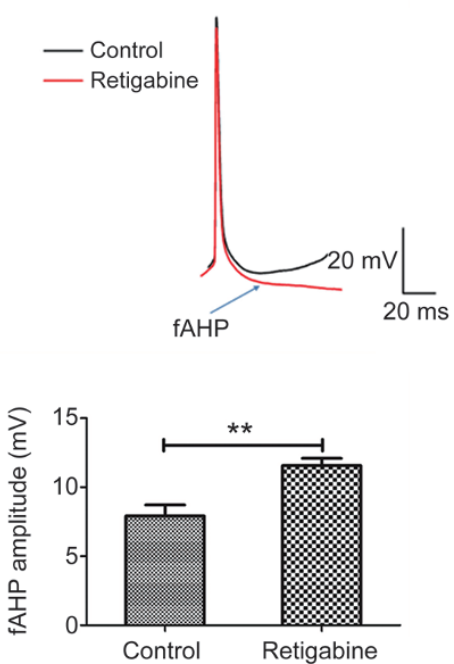
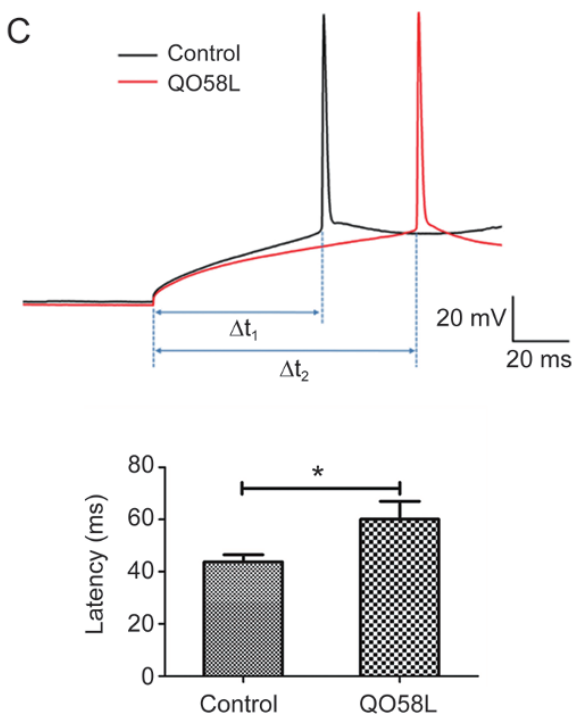

F
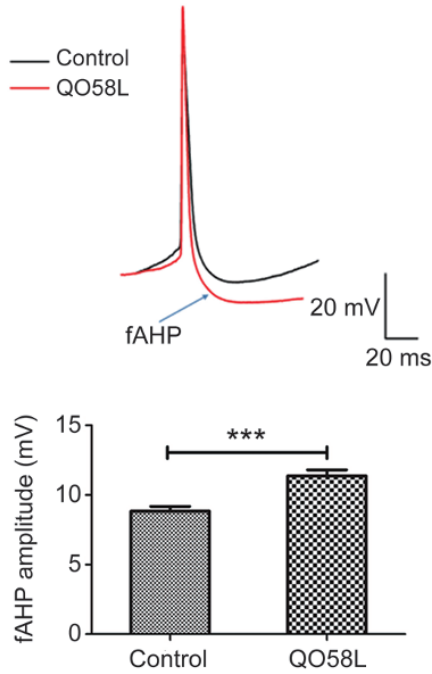

Figure 3. Modulation of the spike onset latency and fAHP by M-channel modulators. (A) Top panel, superimposed traces of the spike onset latencies following the injection of a 100 pA current before and after perfusion with XE991 (40 $\mu \mathrm{mol} / \mathrm{L})$; bottom, bar graph indicating the XE991-induced reduction in the spike onset latency following the injection of a $100 \mathrm{pA}$ current. $\Delta \mathrm{t}_{1}$ represents the latency of the control; $\Delta \mathrm{t}_{2}$ represents the latency in the presence of XE991. (B) Top panel, superimposed traces of the spike onset latencies following the injection of a $160 \mathrm{pA}$ current before and after perfusion with retigabine $(10 \mu \mathrm{mol} / \mathrm{L})$; bottom panel, bar graph indicating the retigabine-induced increase in the spike onset latency. $\Delta \mathrm{t}_{1}$ represents the latency of the control; $\Delta t_{2}$ represents the latency in the presence of retigabine. (C) Top panel, superimposed traces of the spike onset latencies following the injection of a $160 \mathrm{pA}$ current before and after perfusion with the opener Q058L (10 $\mu \mathrm{mol} / \mathrm{L})$; bottom, bar graph indicating the Q058L-induced increase in the spike onset latency. $\Delta \mathrm{t}_{1}$ represents the latency of the control; $\Delta \mathrm{t}_{2}$ represents the latency in the presence of $\mathrm{Q} 058 \mathrm{~L}$. (D) Top panel, superimposed traces of the fAHP amplitudes before and after the application of XE991 (40 $\mu \mathrm{mol} / \mathrm{L})$; bottom, bar graph indicating the XE991-induced reduction of the fAHP amplitude. (E) Top panel, superimposed traces of the fAHP amplitudes before and after the application of retigabine (10 $\mu$ mol/L); bottom, bar graph indicating the retigabine-induced increase in the fAHP amplitude. (F) Top panel, superimposed traces of the fAHP amplitudes before and after the application of Q058L $(10 \mu \mathrm{mol} / \mathrm{L})$; bottom panel, bar graph indicating the Q058L-induced increase in the fAHP amplitude. Data are presented as the mean \pm SEM. $n=7-10$. ${ }^{\star} P<0.05,{ }^{* \star} P<0.01,{ }^{\star \star *} P<0.001$.

excitability. RMP was recorded in neurons in current clamp mode in response to a depolarizing step current $(1 \mathrm{~s}$ duration); the spike frequency increased with increasing input current amplitude in all groups (Figure 2). The application of 40 $\mu \mathrm{mol} / \mathrm{L}$ XE991 resulted in an increase in firing $(n=13, P<0.001$, Figure 2A), whereas the application of the channel opener retigabine at $10 \mu \mathrm{mol} / \mathrm{L}$ or $10 \mu \mathrm{mol} / \mathrm{L}$ QO58L decreased the overall spike frequency for each current input amplitude $(n=5, P<0.001$, Figure 2B; and $n=8, P<0.001$, Figure 2C). Consistent with the spike frequency, the ISI was largely reduced by XE991 (control 129.1 $\pm 11.27 \mathrm{~ms}$ vs XE991 $20.4 \pm 5.75 \mathrm{~ms}, n=6$, $P<0.001$, Figure 2A, bottom). Retigabine and QO58L signifi- 
A

Control

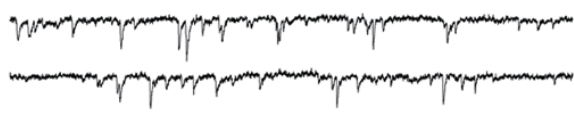

XE991
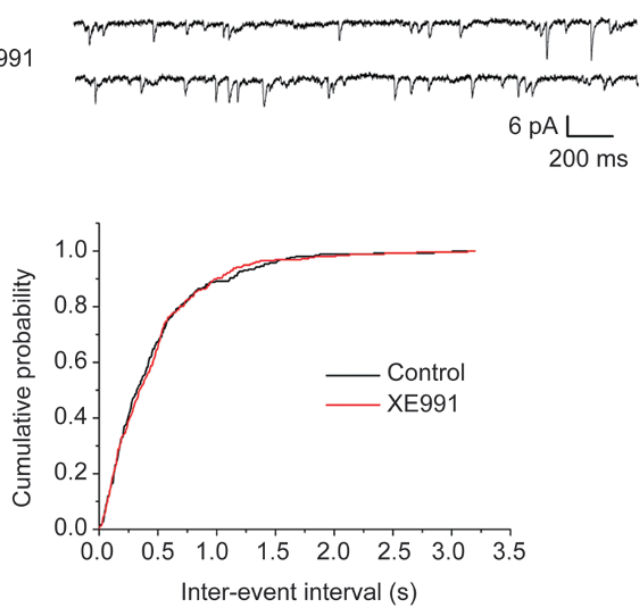

C

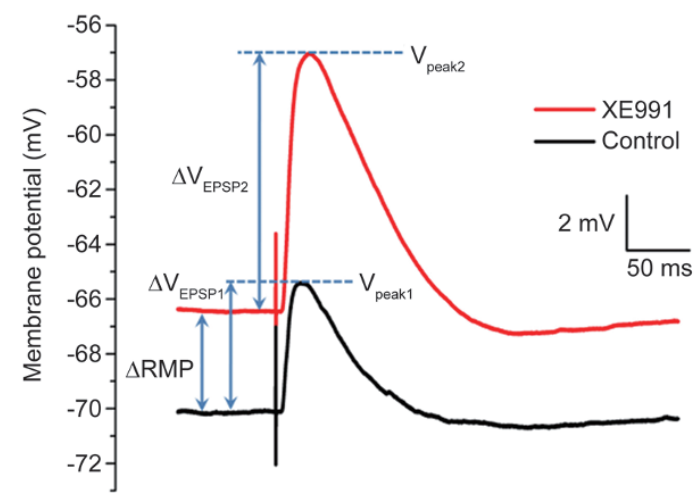

E

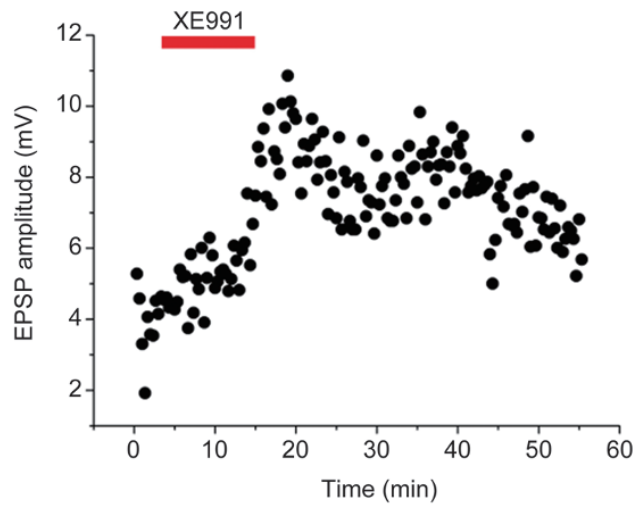

B Control
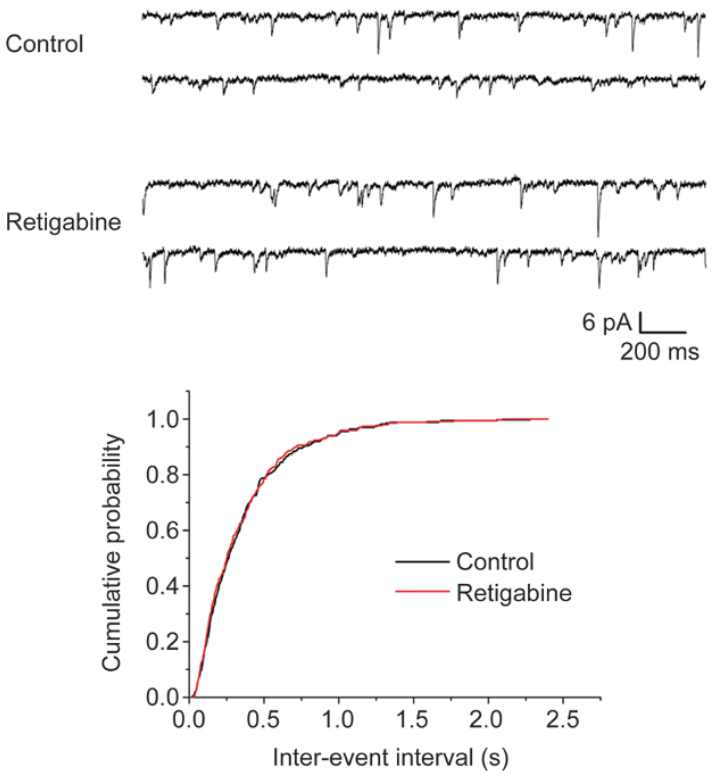

D

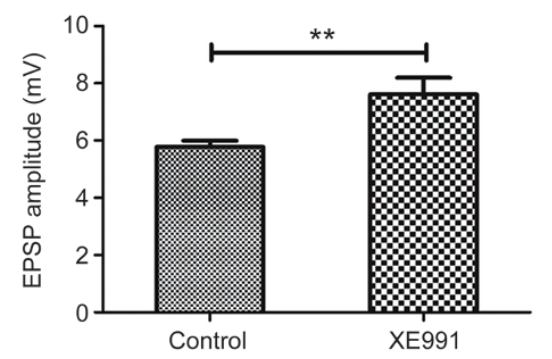

F

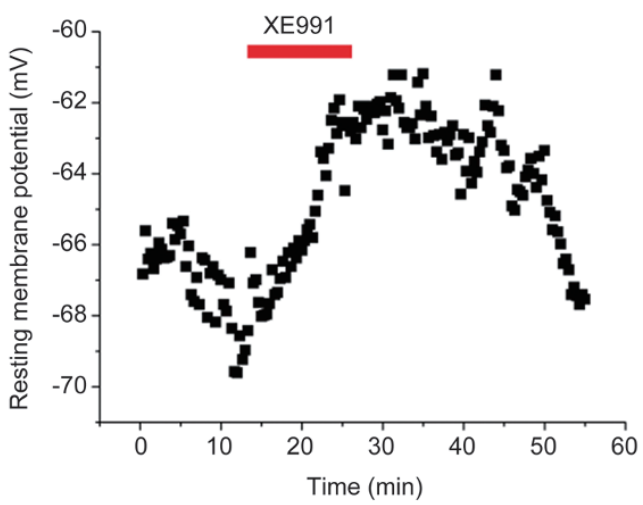

Figure 4. The M-current modulated the post-synaptic excitatory synaptic transmission of PFC layer $\vee$ pyramidal neurons. (A) Top panel, representative traces of mEPSCs observed in the absence and presence of XE991 (40 $\mu \mathrm{mol} / \mathrm{L})$; bottom, cumulative probability graph indicating that $40 \mu \mathrm{mol} / \mathrm{L}$ XE991 had no effect on the mEPSC frequency. (B) Top panel, representative traces of mEPSCs recorded before and after the application of retigabine (10 $\mu \mathrm{mol} / \mathrm{L}$ ); bottom, cumulative probability graph indicating that $10 \mu \mathrm{mol} / \mathrm{L}$ retigabine had no effect on the mEPSC frequency. (C) Representative traces of inter-laminar-evoked single EPSPs before and after the application of XE991 (40 $\mu \mathrm{mol} / \mathrm{L})$. XE991 not only increased the EPSP amplitude $\left(\triangle V_{\text {EPSP }}\right)$ but also depolarized the RMP. (D) Bar graph indicating the XE991-induced increase in the EPSP amplitude. (E) Time course illustrating the increase in the EPSP amplitude in slices perfused with XE991 (40 $\mu \mathrm{mol} / \mathrm{L})$ and following wash-out. (F) Time course illustrating the depolarizing RMP induced by XE991 $(40 \mu \mathrm{mol} / \mathrm{L})$ and repolarization induced by wash-out. Data are shown as the mean \pm SEM. $n=11 .{ }^{* *} P<0.01$. 
cantly increased the ISI (control $85.54 \pm 6.59 \mathrm{~ms}$ vs retigabine $157.2 \pm 15.89 \mathrm{~ms}, n=5, P<0.01$, Figure $2 \mathrm{~B}$, bottom; and control $85.8 \pm 8.55 \mathrm{~ms}$ vs QO58L $181.5 \pm 20.60 \mathrm{~ms}, n=7, P<0.01$, Figure $2 \mathrm{C}$, bottom). Inhibition of M-channels with XE991 induced burst firing in most neurons $(n=10, P<0.001$, Figure 2D). Thus, the intrinsic excitability of PFC neurons is regulated by voltagegated M-channels.

\section{Modulations of the spike onset latency and fAHP amplitude of PFC layer V pyramidal neurons by M-current modulators}

To examine the effect of M-current on firing properties, we determined the effects of the three channel modulators on the spike onset latency and fAHP amplitude (Figure 3). The spike onset latency was analyzed by calculating the time from the onset of the depolarizing current step to the occurrence of the first spike. The application of $40 \mu \mathrm{mol} / \mathrm{L}$ XE991 significantly reduced the latency (XE991 45.07 $\pm 3.24 \mathrm{~ms}$ vs control 71.11 \pm 4.28 ms, $n=10, P<0.001$, Figure $3 \mathrm{~A})$, whereas retigabine $(59.63 \pm 4.94$ $\mathrm{ms} v$ s control $45.81 \pm 2.37 \mathrm{~ms}, n=7, P<0.05$, Figure $3 \mathrm{~B}$ ) or QO58L $(60.07 \pm 6.89 \mathrm{~ms}$ vs control $43.78 \pm 2.70 \mathrm{~ms}, n=10, P<0.05$, Figure 3C) significantly increased the latency. The fast component of the afterhyperpolarization (fAHP) was measured following a single spike in repetitive action potentials. The fAHP amplitude was significantly decreased after perfusion with XE991 (XE991 4.57 $\pm 0.40 \mathrm{mV}$ vs control 8.67 $\pm 0.42 \mathrm{mV}, n=11, P<0.001$, Figure 3D). However, retigabine $(11.57 \pm 0.52 \mathrm{mV}$ vs control $7.92 \pm 0.80 \mathrm{mV}, n=7, P<0.01$, Figure $3 \mathrm{E})$ and QO58L $(11.38 \pm 0.44$ $\mathrm{mV}$ us control $8.84 \pm 0.34 \mathrm{mV}, n=10, P<0.001$, Figure $3 \mathrm{~F}$ ) significantly increased the fAHP amplitude.

\section{Modulation of excitatory synaptic responses of PFC layer V pyramidal neurons by M-current modulators}

In some neurons, M-channels contribute to action potentialindependent glutamatergic synaptic transmission by increasing the mEPSC frequency ${ }^{[23,24]}$. We recorded mEPSCs when neurons were clamped at $-65 \mathrm{mV}$ in the presence of bicuculline $(10 \mu \mathrm{mol} / \mathrm{L})$ and TTX $(0.5 \mu \mathrm{mol} / \mathrm{L})$ with a consistent perfusion of ACSF to determine whether M-channels also regulate pre-synaptic glutamate transmission in PFC layer $\mathrm{V}$ pyramidal neurons. The mEPSC frequency recorded from $\mathrm{mPFC}$ layer $\mathrm{V}$ pyramidal neurons was not affected by either XE991 $(40 \mu \mathrm{mol} / \mathrm{L})$ or retigabine $(10 \mu \mathrm{mol} / \mathrm{L})$ (Figure $4 \mathrm{~A}$ and B). In contrast, the inter-laminar-evoked single EPSP amplitude was significantly increased by XE991 (control $5.78 \pm 0.22 \mathrm{mV}$ vs XE991 7.62 $\pm 0.58 \mathrm{mV}, n=11, P<0.01$, Figure $4 \mathrm{D}$ ). As shown in Figure 4C, XE991 not only increased the EPSP amplitude but also induced the depolarization of RMP, which shifted the $V_{\text {peak }}$ closer to the action potential threshold. In addition, the times when the increasing EPSP amplitude and depolarizing RMP occurred were precisely synchronized, as shown in the time course (Figure 4E and 4F). Thus, M-channels modulate the glutamatergic excitatory synaptic responses of PFC layer $\mathrm{V}$ pyramidal neurons through a post-synaptic mechanism.

\section{Discussion}

The goal of this study was to identify the native voltage-gated
$\mathrm{Kv} 7 / \mathrm{M}$-current and investigate the regulatory effects of pharmacological modulation of the channel on neuronal activities in the PFC. We were able to record the KCNQ/M-currents in layer $\mathrm{V}$ pyramidal neurons from slices of the PFC using a whole-cell patch clamp assay and confirmed the presence of the M-current using specific modulators of the channel. The finding that the selective KCNQ/M-channel blocker XE991 suppressed the KCNQ/M-current, which was increased by the channel selective openers retigabine and QO58L, indicates the functional expression of KCNQ/M-channels in PFC $\mathrm{V}$ layer pyramidal neurons. XE991 induces a depolarizing RMP, increases the average evoked action potential frequency and facilitates burst firing. In contrast, the channel openers retigabine and QO58L decrease the average evoked action potential frequency. Inhibition of the M-current with XE991 also decreases the spike onset latency and the fAHP amplitude, whereas activating the channel with either retigabine or QO58L prolongs the spike onset latency and increases fAHP amplitude. Based on these results, the KCNQ/M-channel modulates the intrinsic excitability of pyramidal neurons in the PFC.

Although KCNQ/M-channels have been reported to modulate mEPSCs in neurons ${ }^{[23,33]}$, the mEPSC frequency was not affected by KCNQ/M-channels in PFC V layer pyramidal neurons in this study. In contrast, XE991 depolarized the RMP, and the amplitudes of single EPSPs evoked in the presence of XE991 were larger than the amplitudes observed in the control condition. Thus, our findings show that the increased excitatory synaptic response induced by KCNQ/M-channel inhibitors was mediated by a post-synaptic mechanism. Taken together, these results reveal a critical role for KCNQ/ M-channels in PFC V layer pyramidal neurons, suggesting an important function of $\mathrm{KCNQ} / \mathrm{M}$-channel in cortical working memory.

A previous study by Cooper et al shows immunofluorescent staining for KCNQ in the PFC and other brain regions ${ }^{[34]}$. The PFC has a well-established role in cognitive information processing for controlling learning, executive functions and working memory ${ }^{[3,8,11,35]}$. PFC dysfunction has been implicated in different psychiatric disorders, such as depression, schizophrenia and bipolar disorder ${ }^{[3,36]}$. As shown in the study by Yuen et al, repeated exposure to stress impairs temporal order recognition memory by suppressing glutamate receptor expression and synaptic transmission ${ }^{[35]}$. According to Wang et al, an age-related decrease in the firing rate of PFC DELAY neurons, which fire during the delay period of working memory, as well as an age-related cognitive decline are partially restored to more youthful levels by blocking $\mathrm{HCN}$ or KCNQ channels ${ }^{[8]}$. Neural excitability and synaptic responses in the PFC were regulated by pharmacological modulation of $\mathrm{KCNQ} / \mathrm{M}$-channels in our study, suggesting that the KCNQ/ $\mathrm{M}$-channel regulates cognitive functions and serves as a potential drug target for the treatment of neuropsychiatric diseases.

Activation of M-channels modulates intrinsic neural excitability by hyperpolarizing RMP and suppressing repetitive action potentials. The two different effects suggest that the 
channel is located in the somatodendrite region and axon hillock $^{[12,22,29,37]}$. In hippocampal CA1 pyramidal neurons, $\mathrm{M}$-channels are critically required for determining the inherent spontaneous firing properties, independent of alterations in synaptic activity ${ }^{[38]}$. In pyramidal neurons of the infralimbic prefrontal cortex, inhibition of M-channels with XE991 increases the number of spikes evoked by a depolarizing pulse and reduces the first interspike interval ${ }^{[18]}$. The expression of KCNQ2/KCNQ3 channels on the surface of axons at both the axonal initial segment and more distal regions suggests that the channel may modulate pre-synaptic neurotransmitter release. In different brain regions, $\mathrm{M}$-channels were reported to modulate the release of glutamate, GABA, $\left[{ }^{3} \mathrm{H}\right] \mathrm{D}$-aspartate and dopamine ${ }^{[23,33,39-41]}$. However, based on our findings, the frequency of mEPSCs was not affected by XE991 or retigabine, suggesting that M-channels in PFC V layer pyramidal neurons are unlikely to be involved in modulating pre-synaptic glutamate release. In contrast, the regulatory effect on glutamatergic synaptic transmission appears to be mediated by a postsynaptic mechanism. Therefore, our data support the hypothesis that the M-current modulates postsynaptic glutamine release, consistent with the observations reported by Shah et al, who showed that only somatic Kv7 channels influence the shape and summation of the synaptic potential ${ }^{[42]}$. The impact of an EPSP on firing does not depend on its amplitude, but on the peak voltage, which determines the suprathreshold of an EPSP ${ }^{[43,44]}$. Our results show that M-channel blockade by XE991 not only increases the evoked EPSP amplitude but also depolarizes the RMP. These two superimposed effects make it easier to trigger an action potential.

In summary, the native $\mathrm{M}$-current regulates the intrinsic excitability and synaptic response of layer $\mathrm{V}$ pyramidal neurons in the PFC. Pharmacological modulation of M-channels in the PFC may provide a therapeutic strategy for neuropsychiatric disorders such as depression and schizophrenia, in which abnormal excitability and synaptic transmission have been observed.

\section{Acknowledgements}

This work was supported by research grants to KeWei WANG from Ministry of Science and Technology of China (No 2013CB531302, 2014ZX09507003-006-004, and 2013ZX09103001-015). We are grateful to lab members Cao $X$, Tang YQ, Ma TY for constructive discussions, and to Ms Li $\mathrm{H}$ for providing assistance with this study. Ke-Wei WANG wishes to thank Wang JM for her consistent support during this study.

\section{Author contribution}

Hui PENG conducted the experiments, analyzed the data and drafted the manuscript; Xi-ling BIAN conducted the experiments; Fu-cui MA assisted with the electrophysiology experiments; Ke-Wei WANG designed the study and finalized the manuscript. All authors read and approved the final manuscript.

\section{References}

1 Fuster JM. Unit activity in prefrontal cortex during delayedresponse performance: neuronal correlates of transient memory. J Neurophysiol 1973; 36: 61-78.

2 Goldman-Rakic PS. The "psychic" neuron of the cerebral cortex. Ann N Y Acad Sci 1999; 868: 13-26.

3 Goldman-Rakic PS. Cellular basis of working memory. Neuron 1995; 14: 477-85.

4 Li M, Long C, Yang L. Hippocampal-prefrontal circuit and disrupted functional connectivity in psychiatric and neurodegenerative disorders. Biomed Res Int 2015; 2015: 810548.

5 Negron-Oyarzo I, Aboitiz F, Fuentealba P. Impaired functional connectivity in the prefrontal cortex: a mechanism for chronic stressinduced neuropsychiatric disorders. Neural Plast 2016; 2016: 7539065.

6 Arnsten AF, Rubia K. Neurobiological circuits regulating attention, cognitive control, motivation, and emotion: disruptions in neurodevelopmental psychiatric disorders. J Am Acad Child Adolesc Psychiatry 2012; 51: 356-67.

7 Faber ES. Functional interplay between NMDA receptors, SK channels and voltage-gated $\mathrm{Ca}^{2+}$ channels regulates synaptic excitability in the medial prefrontal cortex. J Physiol 2010; 588: 1281-92.

8 Wang M, Gamo NJ, Yang Y, Jin LE, Wang XJ, Laubach M, et al. Neuronal basis of age-related working memory decline. Nature 2011; 476: 210-3.

9 Salazar-Villanea M, Liebmann E, Garnier-Villarreal M, MontenegroMontenegro E, Johnson DK. Depressive symptoms affect working memory in healthy older adult hispanics. J Depress Anxiety 2015; 4. pii: 204.

10 Jackson ME, Homayoun H, Moghaddam B. NMDA receptor hypofunction produces concomitant firing rate potentiation and burst activity reduction in the prefrontal cortex. Proc Natl Acad Sci U S A 2004; 101: 8467-72.

11 Yuen EY, Liu W, Karatsoreos IN, Feng J, McEwen BS, Yan Z. Acute stress enhances glutamatergic transmission in prefrontal cortex and facilitates working memory. Proc Natl Acad Sci U S A 2009; 106: 14075-9.

12 Kapfhamer D, Berger KH, Hopf FW, Seif T, Kharazia V, Bonci A, et al. Protein Phosphatase 2a and glycogen synthase kinase 3 signaling modulate prepulse inhibition of the acoustic startle response by altering cortical M-Type potassium channel activity. J Neurosci 2010; 30: 8830-40.

13 Lahti AC, Holcomb HH, Medoff DR, Tamminga CA. Ketamine activates psychosis and alters limbic blood flow in schizophrenia. Neuroreport 1995; 6: 869-72.

14 Breier A, Malhotra AK, Pinals DA, Weisenfeld NI, Pickar D. Association of ketamine-induced psychosis with focal activation of the prefrontal cortex in healthy volunteers. Am J Psychiatry 1997; 154: 805-11.

15 Liston C, Miller MM, Goldwater DS, Radley JJ, Rocher AB, Hof PR, et al. Stress-induced alterations in prefrontal cortical dendritic morphology predict selective impairments in perceptual attentional set-shifting. J Neurosci 2006; 26: 7870-4.

16 Cerqueira JJ, Mailliet F, Almeida OF, Jay TM, Sousa N. The prefrontal cortex as a key target of the maladaptive response to stress. J Neurosci 2007; 27: 2781-7.

17 Drevets WC. Functional anatomical abnormalities in limbic and prefrontal cortical structures in major depression. Prog Brain Res 2000; 126: 413-31.

18 Santini E, Porter JT. M-type potassium channels modulate the intrinsic excitability of infralimbic neurons and regulate fear expression and 
extinction. J Neurosci 2010; 30: 12379-86.

19 Wulff H, Castle NA, Pardo LA. Voltage-gated potassium channels as therapeutic targets. Nat Rev Drug Discov 2009; 8: 982-1001.

20 Wang HS, Pan Z, Shi W, Brown BS, Wymore RS, Cohen IS, et al. KCNQ2 and KCNQ3 potassium channel subunits: molecular correlates of the M-channel. Science 1998; 282: 1890-3.

21 Brown DA, Passmore GM. Neural KCNQ (Kv7) channels. Br J Pharmacol 2009; 156: 1185-95.

22 Chung HJ, Jan YN, Jan LY. Polarized axonal surface expression of neuronal KCNQ channels is mediated by multiple signals in the KCNQ2 and KCNQ3 C-terminal domains. Proc Natl Acad Sci U S A 2006; 103: 8870-5.

23 Sun J, Kapur J. M-type potassium channels modulate Schaffer collateral-CA1 glutamatergic synaptic transmission. J Physiol 2012; 590: 3953-64.

24 Vervaeke K, Gu N, Agdestein C, Hu H, Storm JF. Kv7/KCNQ/

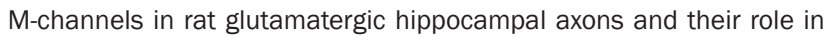
regulation of excitability and transmitter release. J Physiol 2006; 576 : 235-56.

25 Parent MA, Amarante LM, Swanson K, Laubach M. Cholinergic and ghrelinergic receptors and KCNQ channels in the medial PFC regulate the expression of palatability. Front Behav Neurosci 2015; 9: 284.

26 Qi J, Zhang F, Mi Y, Fu Y, Xu W, Zhang D, et al. Design, synthesis and biological activity of pyrazolo[1,5-a]pyrimidin-7(4H)-ones as novel Kv7/KCNQ potassium channel activators. Eur J Med Chem 2011; 46: 934-43.

27 Gulledge AT, Jaffe DB. Dopamine decreases the excitability of layer $\checkmark$ pyramidal cells in the rat prefrontal cortex. J Neurosci 1998; 18: 9139-51.

28 Lamas JA, Selyanko AA, Brown DA. Effects of a cognition-enhancer, linopirdine (DuP 996), on M-type potassium currents $I_{K(M)}$ and some other voltage- and ligand-gated membrane currents in rat sympathetic neurons. Eur J Neurosci 1997; 9: 605-16.

29 Lawrence JJ, Saraga F, Churchill JF, Statland JM, Travis KE, Skinner $\mathrm{FK}$, et al. Somatodendritic Kv7/KCNQ/M channels control interspike interval in hippocampal interneurons. J Neurosci 2006; 26: 1232538.

30 Main MJ, Cryan JE, Dupere JR, Cox B, Clare JJ, Burbidge SA. Modulation of $K C N Q 2 / 3$ potassium channels by the novel anticonvulsant retigabine. Mol Pharmacol 2000; 58: 253-62.

31 Otto JF, Kimball MM, Wilcox KS. Effects of the anticonvulsant retigabine on cultured cortical neurons: changes in electroresponsive properties and synaptic transmission. Mol Pharmacol 2002; 61: 921-7.

32 Zhang F, Mi Y, Qi JL, Li JW, Si M, Guan BC, et al. Modulation of $\mathrm{K}_{\mathrm{v}} 7$ potassium channels by a novel opener pyrazolo[1,5-a]pyrimidin-7(4H)one compound Q0-58. Br J Pharmacol 2013; 168: 1030-42.

33 Peretz A, Sheinin A, Yue C, Degani-Katzav N, Gibor G, Nachman R, et al. Pre- and postsynaptic activation of M-channels by a novel opener dampens neuronal firing and transmitter release. J Neurophysiol 2007; 97: 283-95.

34 Cooper EC, Aldape KD, Abosch A, Barbaro NM, Berger MS, Peacock WS, et al. Colocalization and coassembly of two human brain M-type potassium channel subunits that are mutated in epilepsy. Proc Natl Acad Sci U S A 2000; 97: 4914-9.

35 Yuen EY, Wei J, Liu W, Zhong P, Li X, Yan Z. Repeated stress causes cognitive impairment by suppressing glutamate receptor expression and function in prefrontal cortex. Neuron 2012; 73: 962-77.

36 Arnsten AF. Catecholamine and second messenger influences on prefrontal cortical networks of "representational knowledge": a rational bridge between genetics and the symptoms of mental illness. Cereb Cortex 2007; 17: i6-15.

37 Devaux JJ, Kleopa KA, Cooper EC, Scherer SS. KCNQ2 is a nodal K ${ }^{+}$ channel. J Neurosci 2004; 24: 1236-44.

38 Shah MM, Migliore M, Valencia I, Cooper EC, Brown DA. Functional significance of axonal Kv7 channels in hippocampal pyramidal neurons. Proc Natl Acad Sci U S A 2008; 105: 7869-74.

39 Takahashi Y, Kaba H. Muscarinic receptor type 1 (M1) stimulation, probably through KCNQ/Kv7 channel closure, increases spontaneous GABA release at the dendrodendritic synapse in the mouse accessory olfactory bulb. Brain Res 2010; 1339: 26-40.

40 Luisi R, Panza E, Barrese V, lannotti FA, Viggiano D, Secondo A, et al. Activation of pre-synaptic M-type $\mathrm{K}^{+}$channels inhibits [3H]D-aspartate release by reducing $\mathrm{Ca}^{2+}$ entry through $\mathrm{P} / \mathrm{Q}$-type voltage-gated $\mathrm{Ca}^{2+}$ channels. J Neurochem 2009; 109: 168-81.

41 Jensen MM, Lange SC, Thomsen MS, Hansen HH, Mikkelsen JD. The pharmacological effect of positive KCNQ (Kv7) modulators on dopamine release from striatal slices. Basic Clin Pharmacol Toxicol 2011; 109: 339-42.

42 Shah MM, Migliore M, Brown DA. Differential effects of Kv7 (M-) channels on synaptic integration in distinct subcellular compartments of rat hippocampal pyramidal neurons. J Physiol 2011; 589: 602938.

43 George MS, Abbott LF, Siegelbaum SA. HCN hyperpolarizationactivated cation channels inhibit EPSPs by interactions with M-type $\mathrm{K}^{+}$ channels. Nat Neurosci 2009; 12: 577-84.

44 Koch C, Bernander O, Douglas RJ. Do neurons have a voltage or a current threshold for action potential initiation? J Comput Neurosci 1995; 2: 63-82. 